\title{
Lower Jaw Incisor
}

National Cancer Institute

\section{Source}

National Cancer Institute. Lower Jaw Incisor. NCI Thesaurus. Code C49583.

Either of the incisors found in the mandible. 